\title{
Rouse Dynamics of Polymer Networks Bearing Dendritic Wedges
}

\author{
Andrew A. Gurtovenko, ${ }^{\dagger \neq}$ Yuli Ya. Gotlib, ${ }^{\ddagger}$ and Alexander Blumen*,t \\ Theoretische Polymerphysik, Universität Freiburg, Hermann-Herder-Strasse 3, \\ D-79104 Freiburg, Germany, and Institute of Macromolecular Compounds, \\ Russian Academy of Sciences, Bol shoi Prospect 31, V. O., St. Petersburg, 199004 Russia
}

Received March 21, 2002; Revised Manuscript Received J une 3, 2002

\begin{abstract}
We present a theoretical study of a new class of polymer materials of much recent experimental interest: polymer networks bearing dendritic wedges in the middle of long network strands. We focus on the Rouse dynamics of such cross-linked polymers and perform our study in three steps, considering first single dendritic wedges, then linear chains bearing dendritic wedges (CBDWs), and finally networks formed by end-linked CBDWs. Using analytical and numerical calculations we find that for linear CBDWs increasing the generation number $\mathrm{g}$ of dangling dendritic wedges decreases the storage shear modulus $\mathrm{G}^{\prime}(\omega)$ in the low-frequency domain, i.e., makes the large-scale relaxation more rapid. The zero shear viscosity also decreases with $\mathrm{g}$, indicating a trend opposite to that of dendrimers with small g. Our findings are in qualitative agreement with recent rheological experimental data on side chain dendritic pol ymers. On the contrary, increasing $g$ for dendritic wedges attached to regular networks slows down the low-frequency relaxation; this goes so far as to lead for large $g$ in the intermediate frequency domain to a tendency toward a plateau in $\mathrm{G}^{\prime}(\omega)$. We relate these effects to the presence in CBDW systems of the dangling dendritic wedges, which lower the mobility of the linear chains.
\end{abstract}

\section{Introduction}

Dendrimer macromolecules display a series of unique properties, since they possess a regularly monodisperse branched structure. ${ }^{1-6}$ This regularity makes them very attractive for use as building blocks, to obtain complex polymer materials with new architecture. We mention here exemplarily side chain dendritic polymers, consisting of linear chains with pendant dendritic groups. ${ }^{7-9}$ Recently, even more complex polymer structures were synthesized, namely polyurethane networks bearing dendritic wedges of different generations. ${ }^{10}$ In these networks the dendritic wedges are attached to the middle of each chain which forms the network. ${ }^{10}$ The existence of perfectly branched dangling structures (such as the dendritic wedges) in polymer networks renders such systems very interesting for dynamical studies: One has here the possibility to control directly the amount of sol fraction in the network by varying the generation number (i.e. the size of the dendritic wedges); one may recall that the increase of the size of the dangling dendritic structures decreases the mobility of the elastic network strands.

In this paper we study theoretically the dynamics of networks bearing dendritic wedges and focus on the Rouse description. ${ }^{11,12}$ As is well-known, such an approach is simplified, in that it does not take into account the excluded volume and the hydrodynamic interactions. The excluded volume interactions are of importance especially for the local dynamics of dense polymer structures; as far as the hydrodynamic interactions are concerned, we note that the dendrimers become nondraining spheroids with increasing generation number. ${ }^{13}$ Nonetheless, as a first step to understand how the topology of the complex polymer systems under study affects their dynamical properties, we prefer to

* Author to whom correspondence should be addressed.

† Universität Freiburg.

‡ Russian Academy of Sciences. work in the framework of the Rouse model, while keeping in mind all its restrictions.

The paper is organized as follows. Section 2 is devoted to the general description of the model and to the dynamical features which we are interested in, given that usual mechanical experiments measure the fre quency-dependent components of the complex dynamic modulus $\mathrm{G}^{*}(\omega)$, namely the storage, $\mathrm{G}^{\prime}(\omega)$, and the loss, $\mathrm{G}^{\prime \prime}(\omega)$, moduli. In the following sections we develop in three steps the study of dynamics of polymer networks made out of chains bearing dendritic wedges: In section 3 we consider the Rouse dynamics of a single dendritic wedge of arbitrary functionality and generation number. Section 4 is concerned with the dynamics of un-crosslinked polymer chains bearing dendritic wedges in their middle. Here we reproduce qual itatively some dynamical features of un-cross-linked side chain dendritic polymers, which were found in recent rheological experiments. ${ }^{14}$ The dynamics of polymer networks consisting of endlinked chains bearing dendritic wedges is considered in section 5 . Given that in the end-linked network the mobility of a given chain end is hindered by the other chains at the cross-link point, we consider first the limiting case of a network in which the crosslink points (the chain ends) are fully immobile. Then we turn to the more realistic case we are interested in, namely we consider side chain dendritic polymers crosslinked into a regular network. Section 6 ends the paper with a short summary and conclusions.

\section{The Model}

As mentioned above, we develop our study of the dynamics of polymer networks bearing dendritic wedges in three steps, focusing first on a single dendritic wedge, then on a chain bearing a dendritic wedge (CBDW) attached to its middle monomer, and finally on an endlinked network formed by such CBDWs; see Figure 1. We assume that all these polymer systems consist of beads, connected to each other by elastic springs with 
elasticity constant $K$. This means that we treat the dynamics of the systems under study in the framework of the so-called generalized Gaussian structures ${ }^{15-17}$ (GGS), which represent an extension of the classical Rouse model. ${ }^{11,12}$ All beads of the GGS are subject to the same friction constant $\zeta$ with respect to an effective viscous medium, regardless of the specific GGS structure.

The Langevin equation of motion for the Ith bead of the GGS reads

$$
\xi \frac{\mathrm{d} \mathbf{R}_{1}(\mathrm{t})}{\mathrm{dt}}+\mathrm{K} \sum_{\mathrm{m}=1}^{\mathrm{N}_{\mathrm{tot}}} \mathrm{A}_{\mathrm{Im}} \mathbf{R}_{\mathrm{m}}(\mathrm{t})=\xi \mathbf{w}_{\mid}(\mathrm{t})
$$

where $\mathbf{R}_{\text {l }}(\mathrm{t})$ is the position vector of the Ith GGS bead, $\mathbf{A}=\left\{A_{\mid m}\right\}$ is the connectivity matrix of the given GGS (wedges, CBDWs, networks, etc.), and $\mathrm{N}_{\text {tot }}$ is the total number of beads (monomers) in the GGS under study. The nondiagonal $A_{I m}$ equal $(-1)$ if the lth and mth beads are connected and 0 otherwise; $A_{m m}$ equals the number of bonds emanating from the mth bead. The thermal noise $\xi \mathbf{w}_{\mid}(\mathrm{t})$ is assumed to be Gaussian, with $\left\langle\mathbf{w}_{\mathrm{l}}(\mathrm{t})\right\rangle=$ 0 and $<\mathbf{w}_{\mid \alpha}(\mathrm{t}) \mathbf{w}_{\mathrm{m} \beta}\left(\mathrm{t}^{\prime}\right)>=2 \mathrm{k}_{\mathrm{B}} \mathrm{T} \delta_{\mid \mathrm{m}} \delta_{\alpha \beta} \delta\left(\mathrm{t}-\mathrm{t}^{\prime}\right) / \xi$ (here $\alpha$ and $\beta$ denote the $\mathbf{x}, \mathrm{y}$, and $\mathrm{z}$ directions).

We focus here on the rheological properties of polymer networks bearing dendritic wedges. In typical mechanical experiments the complex (shear) modulus $\mathrm{G}^{*}(\omega)$ is measured as response to a harmonic strain field. ${ }^{18}$ This harmonic strain excitation acts on the macromolecules in solution indirectly, through the solvent. The shear modulus $\mathrm{G}^{*}(\omega)$ is then related to the stress which arises in the system. Theoretically, this stress may be calculated using two different methods. The first method consists of the evaluation of the force per unit area induced by the interactions between the monomers of the polymer and the viscous medium (solvent). The second method is to calculate the additional work performed by the system due to the friction of the monomers against the viscous medium. These methods are widely used standard approaches, and their detailed description can be found elsewhere.12,19

In this way, one finds for structures governed by the Rouse dynamics (GGS) that the storage modulus $\mathrm{G}^{\prime}(\omega)$ and the loss modulus $G^{\prime \prime}(\omega)$ (being the real and the imaginary component of the complex shear modulus $\left.\mathrm{G}^{*}(\omega)\right)$ are given in very dilute solution by ${ }^{12}$

$$
\mathrm{G}^{\prime}(\omega)=\mathrm{C} \frac{1}{\mathrm{~N}_{\text {tot }}} \sum_{\mathrm{i}=2}^{\mathrm{N}_{\text {tot }}} \frac{\left(\omega \tau_{0} / 2 \lambda_{\mathrm{i}}\right)^{2}}{1+\left(\omega \tau_{0} / 2 \lambda_{\mathrm{i}}\right)^{2}}
$$

and

$$
G^{\prime \prime}(\omega)=C \frac{1}{N_{\text {tot }}} \sum_{i=2}^{N_{\text {tot }}} \frac{\omega \tau_{0} / 2 \lambda_{i}}{1+\left(\omega \tau_{0} / 2 \lambda_{i}\right)^{2}}
$$

with $\mathrm{C}=v \mathrm{k}_{\mathrm{B}} \mathrm{T}$, where $v$ is the number of polymer monomers (beads) per unit volume in the GGS under study (wedge, CBDW, etc.); furthermore the $\lambda_{\mathrm{i}}$ are the eigenvalues of the connectivity matrix $\mathbf{A}$ of the given GGS, and $\tau_{0}=\xi / K$. In eqs 2 and 3 the vanishing eigenvalue (denoted by $\lambda_{1}=0$ ) is neglected; it corresponds to the translation of the system as a whole and does not contribute to the moduli. Note that the factor 2 in the relaxation times $\tau_{\mathrm{i}}=\tau_{0} / 2 \lambda_{\mathrm{i}}$ in eqs 2 and 3 arises from the second moment of the displacements involved
A

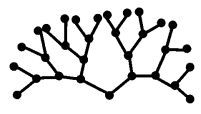

B

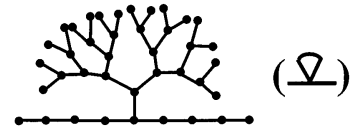

C

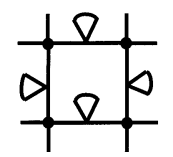

Figure 1. Polymer systems under the study. Shown are (A) a single dendritic wedge, $(B)$ a linear polymer chain bearing a dendritic wedge in its middle (a CBDW), and (C) a regular polymer network bearing dendritic wedges.

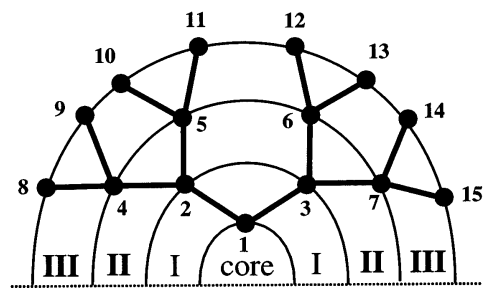

Figure 2. Trifunctional dendritic wedge of third generation, in which the generations and the beads are numbered.

in computing the stress; see the corresponding derivation of the expressions for the moduli in ref 12 .

Moreover, for concentrated solutions, when entanglement effects are negligible (e.g., for polymers of low molecular weight), $\mathrm{G}^{\prime}(\omega)$ and $\mathrm{G}^{\prime \prime}(\omega)$ are still given by eqs 2 and 3 , in which only the prefactor $C$ changes. ${ }^{18}$ Given that we are mostly interested in the slopes of $\mathrm{G}^{\prime}(\omega)$ and $\mathrm{G}^{\prime \prime}(\omega)$, we will present our results in terms of reduced storage and loss moduli, $\left[\mathrm{G}^{\prime}(\omega)\right]$ and $\left[\mathrm{G}^{\prime \prime}(\omega)\right]$, by setting $C=1$ in eqs 2 and 3 .

To conclude this section, one has to emphasize that for calculating the modulus $\mathrm{G}^{*}(\omega)$ it suffices to find only the eigenvalues $\lambda_{i}$ of the connectivity matrix (or the relaxation times $\tau_{i}$ ) of the system under study, since the corresponding eigenfunctions do not enter in eqs 2 and 3. This fact simplifies considerably the solution of dynamical problems for the systems we are interested in.

\section{A Single Dendritic Wedge}

We begin by considering the Rouse dynamics of a single dendritic wedge. If we keep the functionality of all its monomers fixed, the dendritic wedge differs from the usual "full" dendrimer in that its core has one main branch less. In Figure 2 we depict for the functionality $\mathrm{f}=3$ the dendritic wedge with generation number $\mathrm{g}=$ 3. The core (central bead) of the dendritic wedge is numbered by 1 and belongs to generation $\mathrm{g}=0$; in Figure 2 its functionality appears to be $(f-1)$, since in the network it will acquire an additional bond, connecting it to a side chain; see Figure 1.

We remark that we consider here as wedges and "full" dendrimers structures characterized by $\mathrm{f}$ and $\mathrm{g}$ only. More general structures are possible, in which the functionality of the inner branching points, the functionality of the core, and the number of monomers between different branching points can vary. ${ }^{20,21}$ The methods we present here may also be extended to more general situations; we defer such considerations to another work. 
Up to now, several theoretical works were devoted to the theoretical studies of the equilibrium and dynamic properties of dendrimers. ${ }^{20-32}$ In addition, other works concentrated on computer simulations of dendrimers. ${ }^{33-37}$ Most of the existing theoretical studies on the dynamics of dendrimers were based on the Rouse and/or Zimm approaches and focused on the direct diagonalization of the connectivity matrix with the use of analytical $^{22,24,27}$ or numerical methods. ${ }^{28,31,32}$ We remark that solving dynamical problems through the direct analytical diagonalization of the corresponding connectivity matrixes is often quite cumbersome. Recently, one of the authors proposed a new method for finding the ei genvalues (and the corresponding relaxation times) of "full" dendrimers (in the above sense) without a complicated direct diagonalization. ${ }^{38} \mathrm{Here}$ we will apply this approach to dendritic wedges of arbitrary functionality and generation number.

Let us consider a dendritic wedge having as functionality $f$ and as generation number g; see the particular example of Figure 2. The total number of beads in the dendritic wedge, $\mathrm{N}_{\mathrm{w}}$, reads

$$
N_{w}=\frac{(f-1)^{g+1}-1}{f-2}
$$

The method of finding the eigenvalues (the relaxation times) of dendritic wedges is based on simplifying the equations of motion using the topological symmetry of the systems under study. ${ }^{38}$ In this respect the recent work of Cai and Chen ${ }^{27}$ is also very instructive. They studied the Rouse dynamics of dendrimers with trifunctional branching points using the direct analytical diagonalization of the corresponding connectivity matrix and provided a very clear physical interpretation for the relaxation times and the normal modes which they determined. In particular, they found that all types of motions in the dendrimer can be categorized into two general groups: (i) motions involving a mobile core; (ii) motions involving an immobile core. ${ }^{27,38} \mathrm{As}$ we proceed to discuss, the dendritic wedge preserves much of the symmetry of the "full" dendrimer, given that only the functionality of the core is changed. In fact, the eigenvalues of the wedge can be categorized ${ }^{27}$ and evaluated ${ }^{38}$ in the same way as the ones of the corresponding "full" dendrimer.

The description of the method and the detailed evaluation of the eigenvalues and eigenfunctions of dendritic wedges are presented in Appendix A. Here we summarize the results with respect to the eigenvalues. The eigenvalues $\lambda_{k}$ which belong to normal modes with a mobile core have the form of eq A8:

$$
\lambda_{\mathrm{k}}=\mathrm{f}-2 \sqrt{\mathrm{f}-1} \cos \psi_{\mathrm{k}}
$$

Here

$$
\psi_{\mathrm{k}}=\frac{\pi}{\mathrm{g}+1} \mathrm{k} \text { with } \mathrm{k}=1, \ldots, \mathrm{g}
$$

See eq A11. This is a set of g nondegenerate eigenvalues. In addition, one more mode, whose eigenvalue vanishes, $\lambda=0$, belongs to this group; it implies (under the influence of fluctuating forces) the displacement of the dendritic wedge as a whole.

The next group of motions implies an immobile core. In the special case when only the core is immobile, the

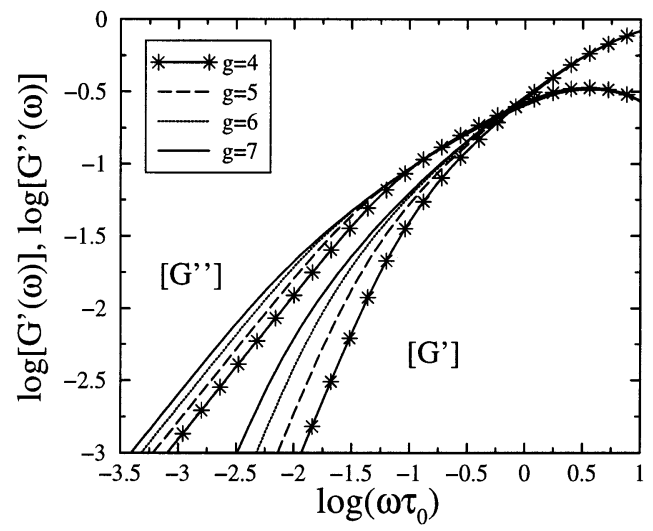

Figure 3. Reduced storage modulus [ $\left.\mathrm{G}^{\prime}(\omega)\right]$ and loss modulus $\left[G^{\prime \prime}(\omega)\right]$ plotted in double logarithmic scales versus the reduced frequency $\omega \tau_{0}$. Shown are results for single dendritic wedges with generation number $g$ ranging from 4 to 7 . The functionality of the wedges, $f$, is equal to 3 .

eigenvalues $\lambda_{k}$ are given again by eq 5 , where the $\psi_{k}$ fulfill

$$
\sin (g+1) \psi_{k}=\sqrt{f-1} \sin g \psi_{k}
$$

See eq A15. It is interesting that eq 7 provides a total of $g$ distinct solutions only if $(g+1)>\sqrt{f-1} g$, i.e., in fact, only in three cases, namely for $(f=3 ; g=1),(f=$ $3 ; g=2)$, and $(f=4 ; g=1)$. In all other cases, i.e., for $(g+1) \leq \sqrt{f-1} g$, eqs 5 and 7 give only $(g-1)$ solutions. Then one additional eigenmode of "exponentialtype" appears, whose eigenvalue has the form of eq A17:

$$
\Lambda=f-2 \sqrt{f-1} \cosh \psi
$$

Here $\psi$ fulfills eq A18:

$$
\sinh (g+1) \psi=\sqrt{f-1} \sinh g \psi
$$

In more general cases, in which larger groups of noncore beads remain immobile, the ei genvalues $\lambda_{k}$ are still given by eq 5 , with $\psi_{\mathrm{k}}$ obeying eq A21:

$$
\sin (\mathrm{g}+1-\mathrm{n}) \psi_{\mathrm{k}}=\sqrt{\mathrm{f}-1} \sin (\mathrm{g}-\mathrm{n}) \psi_{\mathrm{k}}
$$

Here $0<\mathrm{n}<(\mathrm{g}-1)$ and $\mathrm{n}$ denotes the last generation in which all beads are immobile. As in the previous case, eq 10 has $(g-n)$ distinct solutions if $(g-n+1)>$ $\sqrt{f-1}(g-n)$. Otherwise, when $(g-n+1) \leq \sqrt{f-1}(g-$ $n)$, there are $(g-n-1)$ distinct solutions of the form of eq 10 and one has an additional solution in the form of eq 8 with $\psi$ obeying

$$
\sinh (\mathbf{g}-\mathbf{n}+1) \psi=\sqrt{\mathbf{f}-1} \sinh (\mathbf{g}-\mathbf{n}) \psi
$$

See eq A23. Additionally, there is the $(f-1)^{(g-1)}(f-2)$ fold degenerate eigenvalue $\lambda=1$, which corresponds to the motions of the peripheral beads exclusively, $\mathrm{n}=$ $(g-1)$. All in all, as shown in Appendix A, we obtain in this way a total of $N_{w}$ eigenvalues, i.e., all of them.

These findings allow us to study the dynamic properties of dendritic wedges in the GGS framework, given that we derived all the eigenvalues (relaxation times) of such wedges for arbitrary f and g. We are now in the position to use this knowledge to compute the rel axation functions. In Figure 3 we plot the reduced storage, [G'( $\omega)$ ], and loss, [G" $(\omega)$ ], moduli (eqs 2 and 3 with $C=$ 
1) for single dendritic wedges with trifunctional branching points $(f=3)$; in the figure we vary $g$, the generation number, from 4 to 7 . Figure 3 indicates nonscaling forms for $\left[\mathrm{G}^{\prime}(\omega)\right]$ and $\left[\mathrm{G}^{\prime \prime}(\omega)\right]$ in the intermediate frequency domain; i.e., they do not follow power-law, $\omega^{\alpha}$, behaviors. Such nonscaling patterns for [ $\left.\mathrm{G}^{\prime}(\omega)\right]$ and $\left[\mathrm{G}^{\prime \prime}(\omega)\right]$ are typical for dendrimers (see, for instance, ref 31). That this is also here the case follows from the arguments given above, which stressed the close behavior of dendrimers and dendritic wedges. The spectra of a dendrimer and of its corresponding wedge differ only little; only $g$ eigenvalues have different values. The other eigenvalues belong both to the dendrimer and to the wedge and differ only in their degeneracy.

To conclude this section, it is very instructive to estimate the minimal nonvanishing eigenvalue (corresponding to the maximal relaxation time) of the dendritic wedge. We present such an estimation in Appen$\operatorname{dix} B$. It turns out that the minimal, nonvanishing eigenvalue of a dendritic wedge, $\lambda_{\min }$, behaves for large $\mathrm{g} \mathrm{as}^{38}$

$$
\lambda_{\min } \sim \Lambda^{(1)}=\frac{(\mathrm{f}-2)^{2}}{(\mathrm{f}-1)^{(\mathrm{g}+1)}}
$$

See eq B6. This eigenvalue is the same for the "full" dendrimers and for the dendritic wedges because it corresponds to a mode in which the largest dendritic branches move as a whole with respect to each other. ${ }^{27,38}$ In the case $\mathrm{f}=3$, eq 12 leads to the relation $\lambda_{\min } \sim$ $2^{-(g+1)}$, as found by Cai and Chen for dendrimers with trifunctional branching points. ${ }^{27}$

\section{Linear Polymer Chains Bearing Dendritic Wedges}

In this section we focus on un-cross-linked side chain dendritic polymers in solution and study the dynamics of linear polymer chains bearing dendritic wedges (CBDW); see Figure 1B. Again we treat each CBDW as a GGS, so that the CBDW dynamics is again governed by eq 1 . Here we cannot proceed analytically any more and use numerical methods ${ }^{39}$ for the diagonalization of the connectivity matrix $\mathbf{A}$.

The linear chain contains $\mathrm{N}_{\mathrm{ch}}$ beads; for convenience we assume that $\mathrm{N}_{\mathrm{ch}}$ is an odd number, so that a dendritic wedge attached to its central bead divides the chain into two identical halves. The wedge is characterized by the functionality $f$ and by the generation number $\mathrm{g}$ or, alternatively, through eq 4 , by its total number of beads $\mathrm{N}_{\mathrm{w}}$. An interesting question here pertains to the relationship between the sizes of the chain and of the wedge. As it is well-known, 11,12 the longest relaxation time of a Rouse chain, $\tau_{\max }{ }^{(c h)}$, is proportional to $\mathrm{N}_{\mathrm{ch}^{2}}$, where $N_{c h}$ is the number of beads in the chain. The longest relaxation time of a dendritic wedge, $\tau_{\max }(\mathrm{w})$, is for large $g$ proportional to $(f-1)^{g+1}$; see eq 12 . It is evident that in the case of rather short linear chains (i.e. for $\left.\tau_{\max }{ }^{(w)} \approx \tau_{\max }{ }^{(c h)}\right)$ the relaxation of the linear chain will be strongly masked (screened) by the relaxation of the dendritic wedge, especially so because of the high degeneracy of the modes of the wedge in contrast to the situation for linear chains. Thus, the case of rather short linear chains is not interesting, because one can expect here an almost pure dendrimer-like behavior.

We thus will focus on linear chains which are quite long compared to the dimensions of the dangling den-

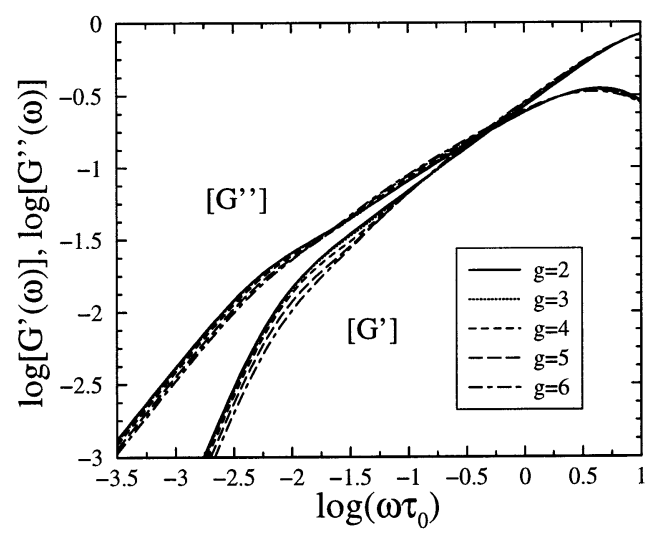

Figure 4. Reduced storage modulus [ $\left.\mathrm{G}^{\prime}(\omega)\right]$ and loss modulus $\left[G^{\prime \prime}(\omega)\right]$ plotted in double logarithmic scales versus the reduced frequency $\omega \tau_{0}$. Shown are results for linear polymer chains of $\mathrm{N}_{\mathrm{ch}}=51$ beads bearing dendritic wedges with $\mathrm{f}=3$ and where g ranges from 2 to 6 .

dritic wedge, i.e., $\tau_{\max }{ }^{(c h)} \gg \tau_{\max }{ }^{(w)}$. This means that the number of beads in the chain $\mathrm{N}_{\mathrm{ch}}$ must be much larger than $(f-1)^{(g+1) / 2}$. In the following we will compare qualitatively our theoretical results with rheological data obtained for side chain dendritic polymers; ${ }^{14}$ in ref 14 the polymers consisted of a polyur ethane main chain and of polyether wedges, of second, third, and fourth generations. In our calculations we prefer to fix the length of the linear chain and to vary for dendritic wedges with $f=3$ the generation number from $g=2$ to $\mathrm{g}=6$. Hence, our linear chain considered must have more than $(f-1)^{(g+1) / 2}=2^{7 / 2} \approx 12$ monomers. In the following we set $\mathrm{N}_{\mathrm{ch}}=51$.

In Figure 4 we present the results of our numerical calculations for the reduced storage, $\left[\mathrm{G}^{\prime}(\omega)\right]$, and loss, [G" $(\omega)$ ], moduli of singlelinear chains $\left(\mathrm{N}_{\mathrm{ch}}=51\right)$ bearing dendritic wedges $(f=3$ and $2 \leq g \leq 6)$. We display both the purely viscous behavior at very low frequencies, where the dynamic moduli obey power laws, ${ }^{12}$ namely $\left[\mathrm{G}^{\prime}(\omega)\right] \sim \omega^{2}$ and $\left[\mathrm{G}^{\prime \prime}(\omega)\right] \sim \omega$ (see eqs 2 and 3 for $\omega \tau_{0} \ll$ $1)$, and also the more interesting, crossover domain at intermediate frequencies. One can see from Figure 4 that in the terminal range of small frequencies $\left[\mathrm{G}^{\prime}(\omega)\right]$ and $\left[G^{\prime \prime}(\omega)\right]$ decrease with increasing g. This result is in qualitative agreement with the experimental data ${ }^{14}$ and can be explained as follows: At high frequencies (i.e. on small length scales) the CBDW dynamics is driven by the relaxation of the dendritic wedge, because of the multiple degeneracy of its relaxation times (eigenvalues), and the relaxation behavior of the linear chain is hidden. In contrast, the CBDW dynamics in the lowfrequency domain is mainly governed by the relaxation of the linear chain. In the case of long linear chains (just what is displayed in Figure 4), the internal relaxation of the wedge is completed on large time scales; in this region the wedge behaves as a "massive" particle having an overall friction constant of $\mathrm{N}_{\mathrm{w}} \zeta$ (one may note that the term "massive" refers here to the friction, since in the Langevin framework, see eq 1, no inertial mass is involved).

In the low-frequency domain the CBDW can thus be viewed as a linear chain with a "massive" particle (in the above sense) attached to it. The friction constant of this particle, $N_{w} \xi$, strongly increases with the generation number $g$ (see eq 4); ther efore, the particle tends to be more and more immobile (damped) with increasing g. This, in turn, hinders more and more the mobility of the central monomer of the chain to which the "massive" 


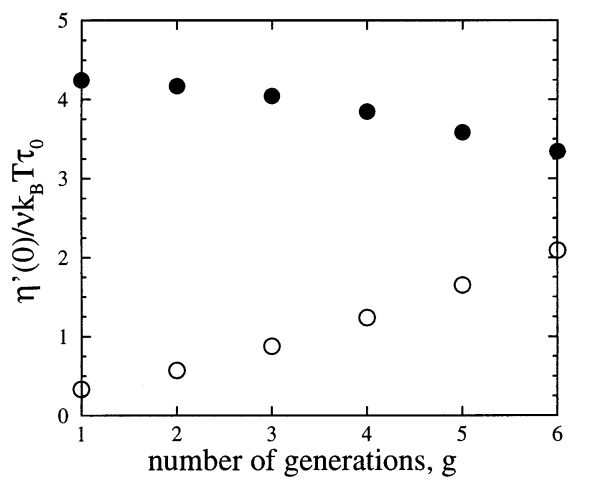

Figure 5. Reduced zero shear viscosity $\eta^{\prime}(0) / \nu \mathrm{k}_{\mathrm{B}} \mathrm{T} \tau_{0}$ plotted as a function of $\mathrm{g}$. Shown are results for single dendritic wedges (open circles) and for the CBDW system given in Figure 4 (filled circles).

particle is attached and ther efore inhibits the excitation of the large-scale asymmetric modes of the chain, leading to a speed-up of the terminal relaxation. As soon as the condition $\tau_{\max }{ }^{(c h)} \gg \tau_{\max }{ }^{(w)}$ holds, the longest CBDW relaxation time gets to be independent of $g$ and equal to the maximal relaxation time of the underlying linear chain, $\tau_{\max }(\mathrm{ch})$. The reason is that for all $\mathrm{g}$ the normal mode related to the longest relaxation time (the smallest nonvanishing eigenvalue) is symmetric with respect to the central bead to which the wedge is attached; it describes the stretching of the chain at its ends, so that the central bead stays immobile; see e.g. ref 40 . Furthermore, we note from Figure 4 that the storage modulus, $\left[\mathrm{G}^{\prime}(\omega)\right]$, is more sensitive to $g$ than the loss modulus, [G" $(\omega)]$.

In continuation we evaluate numerically, for very dilute solutions, the zero shear viscosity $\eta^{\prime}(0)$. In general the shear viscosity $\eta^{\prime}(\omega)$ is related to $\mathrm{G}^{\prime \prime}(\omega)$ through $\eta^{\prime}(\omega)$ $=\mathrm{G}^{\prime \prime}(\omega) / \omega$; see e.g. ref 18. From eq 3 it follows that

$$
\eta^{\prime}(0)=v \mathrm{k}_{\mathrm{B}} \mathrm{T} \frac{1}{\mathrm{~N}_{\text {tot }}} \sum_{\mathrm{i}=2}^{\mathrm{N}_{\text {tot }}} \frac{\tau_{0}}{2 \lambda_{\mathrm{i}}}=v \mathrm{k}_{\mathrm{B}} \mathrm{T} \frac{1}{\mathrm{~N}_{\text {tot }}} \sum_{\mathrm{i}=2}^{\mathrm{N}_{\text {tot }}} \tau_{\mathrm{i}}
$$

Now $\eta^{\prime}(0)$ for side chain dendritic polymers was determined experimentally (see ref 14); there $v$ stood for our $v / \mathrm{N}_{\text {tot. }}$. From eq $13 \eta^{\prime}(0)$ is proportional to the sum of all the finite relaxation times of the system under study. In Figure 5 we plot our numerical results of $\eta^{\prime}(0)$. We consider both single dendritic wedges with $f=3$ and $g$ $=1,2, \ldots, 6$ and linear chains $\left(\mathrm{N}_{\mathrm{ch}}=51\right)$ with such wedges. In the GGS framework used here we find that in the $g$ range considered the $\eta^{\prime}(0)$ of dendritic wedges increases with g. However, for linear CBDW we find the opposite trend, namely that $\eta^{\prime}(0)$ decreases with g; see Figure 5. This stems from the fact that the main contribution to $\eta^{\prime}(0)$, see eq 13 , is due to the long relaxation times (or, stated differently, to the small eigenvalues). Since in the low-frequency domain the relaxation gets to be more rapid with increasing $\mathrm{g}$, the corresponding long relaxation times $\tau_{\mathrm{i}}$ decrease with $\mathrm{g}$, so that $\eta^{\prime}(0)$ also decreases. This effect is indeed confirmed by rheological experiments ${ }^{14}$ on side chain dendritic polymers, with $2 \leq \mathrm{g} \leq 4$.

\section{End-Linked Polymer Networks Bearing Dendritic Wedges}

In this section we consider the dynamics of polymer networks, obtained by cross-linking CBDWs. We focus on end-linked networks; i.e., the CBDWs are cross-

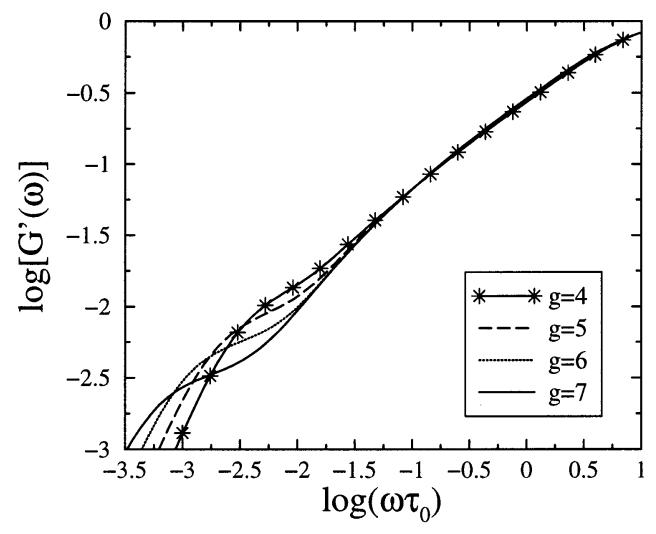

Figure 6. Reduced storage modulus [ $\left.\mathrm{G}^{\prime}(\omega)\right]$ plotted in double logarithmic scales versus the reduced frequency $\omega \tau_{0}$. Shown are results for linear polymer chains (of $\mathrm{N}_{\mathrm{c}}=51$ beads), whose ends are fixed; the chains carry attached wedges with $f=3$ and $g$ ranging from 4 to 7 .

linked at the chain's ends (see Figure 1C). Through the linkage into the network, the ends of the CBDWs loose part of their mobility, since their motion gets hindered by the other CBDWs.

Fixed-Chain-Ends Model. At first, we consider a limiting case, namely a single CBDW, whose chain ends are fixed. Physically, it corresponds to the freezing of the motion of the cross-links (e.g., because the time scales of motion of the network chains and of the crosslinks separate 41,42$)$. As before, we treat the dynamics of such a CBDW through the direct numerical diagonalization of the corresponding connectivity matrix $\mathbf{A}$; see eq 1 . Now, following the ideas of Mooney, ${ }^{18,42}$ the ends of the chain are attached by additional links (springs) to fixed points: this increases the corresponding two diagonal elements of $\mathbf{A}$ by 1 . The new connectivity matrix has then $\mathrm{N}_{\text {tot }}$ positive eigenvalues, and the sums in eqs 2 and 3 run over all of them. In Figure 6 we plot [G' $(\omega)$ ] for CBDWs with fixed ends, whose chains have length $\mathrm{N}_{\mathrm{ch}}=51$ and whose wedges have $\mathrm{f}=3$; furthermore we let for the wedges $g$ range between 4 and 7 . These results can be compared directly to those for uncross-linked CBDWs; see Figure 4. First we observe for $\log \left(\omega \tau_{0}\right)>-2$ that the behavior for free CBDWs and for CBDWs with fixed ends is very similar: this means that in this range of frequencies fixing the chain's ends does not affect the dynamics. On the other hand, at lower frequencies the [ $\mathrm{G}^{\prime}(\omega)$ ] decay becomes slower for cross-linked CBDWs than for free CBDWs. This leads for large $\mathrm{g}$ even to a tendence of forming a plateau around $\log \left(\omega \tau_{0}\right) \sim-2.5$; see Figure 6 . The width of this quasi-plateau region increases with increasing $g$, so that the terminal, $\omega^{2}$ decay of $\left[\mathrm{G}^{\prime}(\omega)\right]$ shows up at lower frequencies for larger g; see Figure 6.

We compare these results to our model of a linear chain with fixed ends and a "massive" particle attached to its center; see Figure 7. Our numerical results are given in Figure 8, where we plot the $\left[\mathrm{G}^{\prime}(\omega)\right.$ ] curves by taking in eq $2 \mathrm{~N}_{\text {tot }}=\mathrm{N}_{\mathrm{w}}+\mathrm{N}_{\mathrm{ch}}$ both for the CBDW with fixed ends and for the chain with the "massive" particle. For long linear chains and low frequencies we expect $\left[\mathrm{G}^{\prime}(\omega)\right]$ to follow the trend of Figure 6 , since at small $\omega$ the relaxation of the chain is still in progress when the wedge's relaxation has ended. As can be inferred by comparing Figures 6 and 8, this is borne out nicely. Furthermore, our simplified model reproduces quite well the flattening of $\left[\mathrm{G}^{\prime}(\omega)\right]$ found for linear CBDWs with fixed ends. 


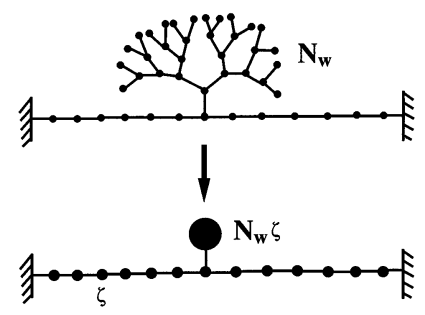

Figure 7. Simplified model proposed to reproduce the terminal region of the relaxation found in Figure 6: a linear chain with fixed ends, to which a "massive" particle is attached. See text for details.

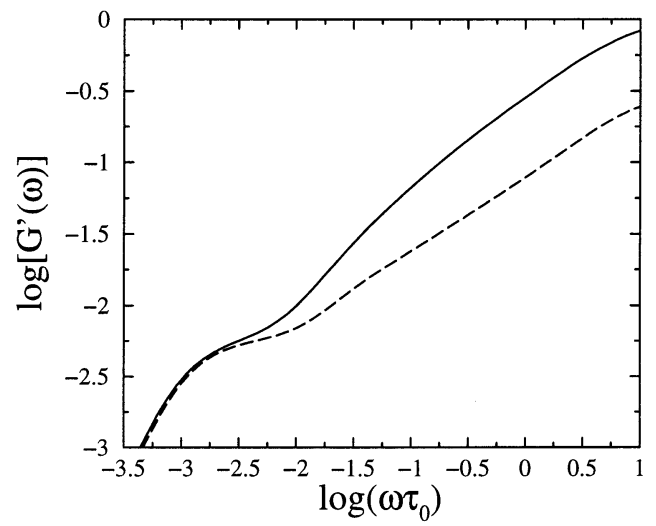

Figure 8. Reduced storage modulus $\left[\mathrm{G}^{\prime}(\omega)\right]$ plotted in double logarithmic scales versus the reduced frequency $\omega \tau_{0}$. Shown are results for a linear CBDW whose ends are fixed (solid line). The results are compared to those for the model of Figure 7 (dashed line). Here $\mathrm{N}_{\mathrm{ch}}=51, \mathrm{f}=3$, and $\mathrm{g}=6$.

The tendency of $\left[G^{\prime}(\omega)\right.$ ] to form a plateau, see Figure 6 , implies a very slow relaxation in the corresponding frequency domain. After most internal relaxation processes are finished, there remain a few modes connected tolarge-scale motion of the center of mass of the CBDW with fixed chain ends. N ote that now, due to the fixing of the ends, its relaxation time is finite (i.e. $\lambda_{1} \neq 0$ ), which is in contrast to the un-cross-linked situation. F or large $g$ the low-frequency relaxation of the linear chain in the CBDW is dominated by the presence of an almost immobile, "massive" dendritic wedge $\left(\mathrm{N}_{\mathrm{w}} \gg 1\right)$. N ow this relaxation ends at $\tau_{\max }{ }^{(\mathrm{ch})} / 4$, where $\tau_{\max }{ }^{(\mathrm{ch})}$ is the maximal relaxation time of the chain. Note that, in contrast to the case of free CBDWs (discussed in the previous section), the fixing of the chain's ends leads here (given that the central monomer is almost immobile) to a lowfrequency relaxation determined by the two (independent) halves of the original chain. An even slower relaxation (the terminal one) involves also the center of mass of the CBDW, so that the "massive" wedge also participates in this motion; this introduces an additional term of $\mathrm{N}_{w} \zeta$ in the global friction involved and, therefore, in the corresponding relaxation times. We hence relate the very interesting, flattening feature found in F igure 6 to the difference between the longest relaxation time of the whole CBDW system, $\tau_{\max }$, and the longest relaxation time of half of the underlying linear chain involved, $\tau_{\max }{ }^{(\mathrm{ch})} / 4$.

End-Linked Regular Networks of CBDWs. In the subsection above we considered the limiting case of CBDWs with fixed ends, corresponding to a network with fully immobile cross-links. Under realistic conditions the mobility of the cross-links is certainly intermediate between completely fixed and completely free. In this way the cross-links lead to large-scale, coopera- tive modes. Such collective relaxation phenomena are less pronounced in un-cross-linked polymers, wherethey may arise due to entangl ements. To depict the real istic situation of mobile cross-links, we proceed following a model previously studied by us; ${ }^{43,44}$ it is based on a regular lattice decorated with complex subunits. Here we take the subunits to be CBDWs; see Figure $1 \mathrm{C}$.

The motivation of the model of refs 43 and 44 (see also ref 45) was to study the dynamics of disordered, highly inhomogeneous polymer networks. The model reflects two aspects often found in such networks, namely that the local structure is rather disordered, whereas the large-scale structure is rather homogeneous: one hence builds the network from subunits, which are connected into a regular structure (say a 2D square lattice). The inner structure of the subunits can be then varied; for ease of computation, however, each realization of the disordered network utilizes a (possibly random, but once chosen, unique) subunit, repeated indefinitely. The regularity of this model allows one then to simplify the problem of finding the eigenvalues (i.e. the relaxation times) of the network. The main idea is that the Langevin equations for such GGSs have a topological symmetry mathematically very akin to the translational symmetry of perfectly regular lattices. ${ }^{43,44}$ We are therefore able to reduce the determination of the normal modes of the whole network to the diagonalization of relatively small matrixes. These matrixes are defined by the topology of the repeating elements (subunits) of the lattice; the large-scale symmetry is taken into account by phase factors corresponding to appropriately chosen (periodic) boundary conditions. ${ }^{43,44}$

We now return to our cross-linked CBDWs system and model it in the spirit expressed above, by taking the CBDWs to be the subunits (building blocks) of a regular lattice. We have now to choose a lattice, and we hence opt for 2D square networks formed from CBDWs (see Figure 1C). We recall that such networks are 2D only in the sense of the connectivity of the crosslinks; the networks move in the 3D Cartesian spacelike fishing nets in water. The question is in how far our results are influenced by the choice of the lattice; it turns out that the lattice influences only the lowfrequency domain, where for instance one has $\left[\mathrm{G}^{\prime}(\omega)\right]$ $\sim \omega^{3 / 2}$ for three-dimensional cubi $c^{46,47}$ and $\left[\mathrm{G}^{\prime}(\omega)\right] \sim \omega$ for two-dimensional square $\mathrm{e}^{48,49}$ networks. On the other hand, the most interesting relaxation effects occur in a frequency domain which is intermediate between the intra-subunit modes and the lattice modes, as previously shown by us $^{43,44}$ in the case when the subunits are "small-world-networks".

In Figure 9 we present the results of our numerical calculations for 2D networks ( $20 \times 20$ cross-links) built from CBDWs. Here we took $\mathrm{N}_{\mathrm{ch}}$, the number of monomers in the linear chains between the cross-links, to be 51 ; we also set $f=3$ for the dendritic wedges. To have a more pronounced effect, we consider dendritic wedges of large generation numbers and take $\mathrm{g}$ to be 5,6 , and 7. As can be seen in Figure 9 , the relaxation features observed in the previous subsection for CBDWs with immobile cross-links carry over to the regularly endlinked networks considered here. F or large g we again find that $\left[\mathrm{G}^{\prime}(\omega)\right.$ ] shows a tendency to form a plateau at intermediate frequencies; this behavior is followed at low frequencies by the power-law relaxation due to the lattice. As mentioned above, such a plateaulike domain stems from a low number of modes in the relaxation 


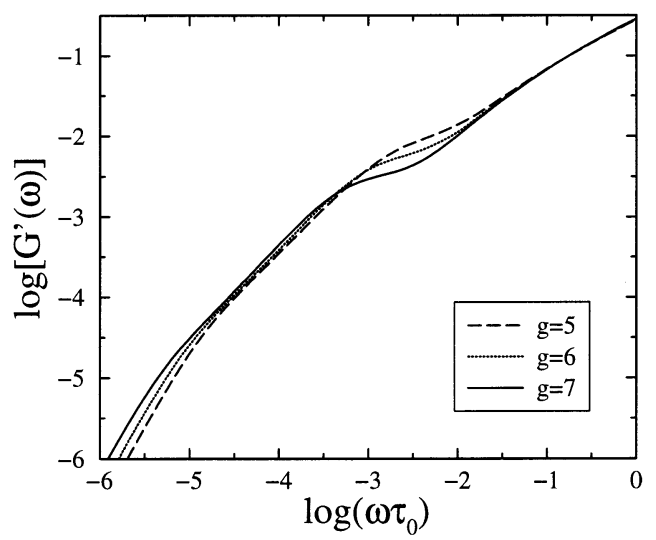

Figure 9. Reduced storage modulus [ $\left.\mathrm{G}^{\prime}(\omega)\right]$ plotted in double logarithmic scales versus the reduced frequency $\omega \tau_{0}$. Shown are results for $2 \mathrm{D}$ regular polymer networks $(20 \times 20$ crosslinks) bearing dendritic wedges of the fifth, sixth, and seventh generations. Here $\mathrm{N}_{\mathrm{ch}}=51$ and $\mathrm{f}=3$.

spectrum of CBDWs with fixed ends; this domain extends from the longest relaxation time of half of the linear chain, $\tau_{\max }{ }^{(\mathrm{ch})} / 4$, to that of the same chain bearing a dendritic wedge, $\tau_{\max }$. This picture is very different from that encountered for regular networks built from linear Rouse chains: in such a situation the longest relaxation times $\tau_{\max }{ }^{(c h)}$ of the chains between cross-links is equal to the minimal relaxation time of the regular lattice. As a result, the relaxation spectrum is continuous and no plateau appears. ${ }^{46-49}$ Hence, the intermediate plateau-type behavior found in Figure 9 is due to the large dangling structures (wedges), which considerably slow the relaxation at intermediate frequencies.

We conclude with some remarks on the low-frequency relaxation behavior, which in Figure 9 sets in at around $\omega \tau_{0} \simeq 10^{-3.5}$. In this region $\left[\mathrm{G}^{\prime}(\omega)\right]$ is determined by the large-scal e connectivity of the network; here it displays the power-law behavior $\left[G^{\prime}(\omega)\right] \sim \omega$, typical for $2 D$ lattices. Now, given the disorder found in randomly cross-linked networks, the relaxation character imposed here by the choice of a 2D regular lattice is debatable; experimentally $\left[G^{\prime}(\omega)\right]$ may well show up a different form at low frequencies. On the other hand, a precise analysis of $\left[\mathrm{G}^{\prime}(\omega)\right]$ at such frequencies requires a good knowledge of the topological, large-scale network structure, combined with very careful measurements in a frequency domain of difficult access, both aspects being worth further studies in the future.

\section{Conclusions}

In this paper we presented a theoretical study of a new class of polymer materials, namely polymer networks bearing dendritic wedges. ${ }^{10}$ In such networks the mobility of the elastic network strands is directly affected by changes in the generation number of the dangling dendritic structures. Given that our goal was to determine the influence of the dendritic wedges on the mechanical characteristics (such as the storage and the loss moduli) of the networks to which they are attached, we concentrated here on the Rouse dynamics of such cross-linked polymers. We performed our study in three steps, considering sequentially single dendritic wedges, linear chains bearing dendritic wedges (CBDWs), and end-linked polymer networks formed by such CBDWs.

On the basis of the previous works in the field, ${ }^{27,38}$ we calculated analytically the set of relaxation times for single dendritic wedges of arbitrary functionality $f$ and generation number $\mathrm{g}$. In particular we showed that the relaxation spectrum of the dendritic wedge differs from that of its related, "full" dendrimer only in the values of $g$ distinct relaxation times; the corresponding modes involve namely all the monomers of the structure and differ in symmetry between wedge and dendrimer. All other relaxation times are the same both for the dendritic wedge and for the dendrimer, the difference residing only in the degeneracy of these modes.

Using numerical methods, we then calculated the dynamics of linear CBDWs. We found that increasing the size of the associated dangling dendritic structures decreases the dynamic shear modulus in the lowfrequency domain, i.e., renders the large-scale relaxation of the CBDWs more rapid. The CBDW's zero shear viscosity also decreases with $\mathrm{g}$, a trend opposite to that of dendrimers with small $\mathrm{g}$. These findings are in qualitative agreement with recent rheological data on side chain dendritic polymers. ${ }^{14}$ Physically these features are due to the fact that a "massive" dendritic wedge hinders the mobility of the central monomer of the linear chain, inhibiting the large-scale normal modes of the chain which are asymmetric with respect to the chain's center.

On the contrary, increasing g slows down the lowfrequency relaxation of CBDWs cross-linked into regular networks. Furthermore, for g large a tendency toward a plateau behavior shows up in the intermediate frequency domain of the storage modulus $\mathrm{G}^{\prime}(\omega)$. In this domain the networks' dynamics is mainly determined by the internal relaxation of the chains with wedges. Due to the low mobility of the chains' ends (which are hindered by the network), the relaxation spectrum of CBDWs networks has only very few modes in the range extending between the longest relaxation time of the underlying linear chain and that of the corresponding CBDWs with fixed ends.

Acknowledgment. Helpful discussions with Dr. A. V. Lyulin are gratefully acknowledged. This work was supported by the Fonds der Chemischen Industrie, by the DFG, and by the INTAS (Grant 00-712). A.A.G. acknowledges the support of the Alexander von Humboldt Foundation. Yu.Ya.G. acknowledges the support of the Russian Federal Program "Integration" (Grant 32638), of the Russian Foundation of Basic Research (Grant 02-03-33132), and of the ESF Scientific Program SUPERNET.

\section{Appendix A}

Here we present the determination of the eigenvalues and eigenfunctions of dendritic wedges.

(i) Mobile Core. When the central bead (core) is mobile, all the beads of the dendrimer are involved in the motion. ${ }^{27,38}$ On the basis of the topological architecture of the dendritic wedge (each inner bead is connected with one bead from the previous generation and $(f-1)$ beads from the next generation), the Langevin equations of motion for the inner beads can be rewritten as follows (cf. eq 1):

$$
\xi \frac{\mathrm{d} \mathbf{R}_{\mathrm{j}, \mathrm{m}}(\mathrm{t})}{\mathrm{dt}}+\mathrm{K}\left[\mathrm{f} \mathbf{R}_{\mathrm{j}, \mathrm{m}}(\mathrm{t})-\mathbf{R}_{\mathrm{j}-1, \mathrm{n}}(\mathrm{t})-\sum_{\mathrm{l}=1}^{\mathrm{f}-1} \mathbf{R}_{\mathrm{j}+1, \mathrm{l}}(\mathrm{t})\right]=0
$$

Here $\mathbf{R}_{\mathrm{j}, \mathrm{m}}(\mathrm{t})$ is the position vector of the $\mathrm{mth}$ bead of 
generation $\mathrm{j}$, where the index $\mathrm{j}$ for the inner beads lies in the range $0<\mathrm{j}<\mathrm{g}(\mathrm{j}=0$ corresponds to the core), and $\mathbf{R}_{\mathrm{j}-1, \mathrm{n}}(\mathrm{t})$ and $\mathbf{R}_{\mathrm{j}+1, \mathrm{I}}(\mathrm{t})(\mathrm{I}=1, \ldots, \mathrm{f}-1)$ correspond to the locations of the nearest neighbors to $\mathbf{R}_{\mathrm{j}, \mathrm{m}}(\mathrm{t})$. We have simplified eq $A 1$ by setting zero on its right-hand side. The justification is that we are interested only in linear relations involving the normal modes; in these terms any dependence on the $\mathbf{w}_{l}(\mathrm{t})$ disappears after thermal averaging.

To solve eq A1 we use a normal mode treatment ${ }^{12,46,47}$ and look for solutions having the form of sums of normal modes $P_{k}(j, m ; t)$, which have a simple time behavior $P_{k}(j, m ; t)=\Pi_{k}(j, m) \exp \left[-\lambda_{k} t / \tau_{0}\right]$ :

$$
\mathbf{R}_{j, m}(\mathrm{t})=\sum_{k} \mathbf{C}_{k} P_{k}(j, m ; t)=\sum_{k} \mathbf{C}_{k} \Pi_{k}(j, m) \exp \left[-\lambda_{k} t / \tau_{0}\right]
$$

Here $\tau_{0}=\xi / \mathrm{K}$ is the characteristic relaxation time of the system, $\mathbf{C}_{\mathrm{k}}$ are j-independent constants, $\lambda_{\mathrm{k}}$ are the eigenvalues, and $\Pi_{k}(j, m)$ are the eigenfunctions of eq $\mathrm{A} 1$. The relaxation times $\tau_{\mathrm{k}}$ are uniquely determined by the eigenvalues $\lambda_{\mathrm{k}}$ through $\tau_{\mathrm{k}}=\tau_{0} / \lambda_{\mathrm{k}}$. Inserting eq A2 into eq A1 leads to

$$
\begin{aligned}
\left(-\lambda_{k}\right) \Pi_{k}(j, m)+\left[f \Pi_{k}(j, m)-\right. & \Pi_{k}(j-1, n)- \\
& \left.\sum_{l=1}^{f-1} \Pi_{k}(j+1, l)\right]=0
\end{aligned}
$$

We proceed now under the key assumption that the normal modes can be characterized by motions involving one "root" bead and all of its descendants of higher generations. For such a subwedge having as ancestor the "root", beads which bel ong to the same generation move in the same manner. 38 Thus, if the central core moves, i.e., is the "root" one has $\Pi_{k}(j, m)=\Pi_{k}(j)$ for all the beads of generation $j$, see ref 38 and also Figure 3 of ref 27. The justification for the key assumption is that it indeed allows us to find all the eigenvalues. Using it eq Al simplifies to

$$
\begin{array}{r}
\left(-\lambda_{k}\right) \Pi_{k}(j)+\left[f \Pi_{k}(j)-\Pi_{k}(j-1)-\right. \\
\left.(f-1) \Pi_{k}(j+1)\right]=0
\end{array}
$$

Note that one has immediately $\Pi_{k}(j)=$ constant and $\lambda_{k}$ $=0$ as a solution of eq A4; this zero eigenvalue $\lambda=0$ implies (under the influence of fluctuating forces) the displacement of the dendritic wedge as a whole. For the remaining modes it is convenient to symmetrize eq $A 4$ using the substitution $\Pi_{k}(j)=(f-1)^{-\mathrm{j} / 2} \Phi_{k}(\mathrm{j})$; one attains the following form: ${ }^{38}$

$$
\begin{aligned}
&\left(-\lambda_{k}\right) \Phi_{k}(j)+f \Phi_{k}(j)-\sqrt{f-1} {\left[\Phi_{k}(j+1)+\right.} \\
&\left.\Phi_{k}(j-1)\right]=0
\end{aligned}
$$

Equation A5 holds for all inner beads, $0<j<g$. The symmetrized equations of motion at the wedge's boundaries, i.e., for $\mathrm{j}=\mathrm{g}$ (peripheral beads) and for $\mathrm{j}=0$ (core), are

$$
\left(-\lambda_{k}\right) \Phi_{k}(g)+\Phi_{k}(g)-\sqrt{f-1} \Phi_{k}(g-1)=0
$$

and

$$
\left(-\lambda_{k}\right) \Phi_{k}(0)+(f-1) \Phi_{k}(0)-\sqrt{f-1} \Phi_{k}(1)=0
$$

Note that the equation of motion for the core, eq A7, represents the only distinction between the "full" dendrimer ${ }^{38}$ and the dendritic wedge considered here.

As it will be demonstrated below, the eigenfunctions $\Phi_{k}(j)$ turn out to be linear combinations of the functions $\Phi_{k}{ }^{c}(j)=\cos \mathrm{j} \psi_{k}$ and $\Phi_{k}{ }^{s}(j)=\sin \mathrm{j} \psi_{k}$, where $\psi_{k}$ play the role of phase shifts. It is now a simple matter to verify that $\Phi_{\mathrm{k}} \mathrm{c}(\mathrm{j})$ and $\Phi_{\mathrm{k}}{ }^{\mathrm{s}}(\mathrm{j})$ satisfy eq $\mathrm{A} 5$ for the eigenvalue: ${ }^{38}$

$$
\lambda_{\mathrm{k}}=\mathrm{f}-2 \sqrt{\mathrm{f}-1} \cos \psi_{\mathrm{k}}
$$

Equations $A 6$ and $A 7$ fix now the form of $\Phi_{k}(j)$. It is namely easy to check that the linear combination

$$
\begin{aligned}
\Phi_{k}(j)=\left(\sqrt{f-1} \cos \psi_{k}-1\right) \Phi_{k}{ }^{s}(j)+ \\
\sqrt{f-1} \sin \psi_{k} \Phi_{k}{ }^{c}(j)
\end{aligned}
$$

i.e.

$$
\Phi_{\mathrm{k}}(\mathrm{j})=\sqrt{\mathrm{f}-1} \sin (\mathrm{j}+1) \psi_{\mathrm{k}}-\sin \mathrm{j} \psi_{\mathrm{k}}
$$

solves eqs A6 and A7 when $\psi_{k}$ has the form

$$
\psi_{\mathrm{k}}=\frac{\pi}{\mathrm{g}+1} \mathrm{k} \text { with } \mathrm{k}=1, \ldots, \mathrm{g}
$$

As a result we have determined through eqs $A 8$ and All g linearly independent solutions for which $\Phi_{k}(0) \neq$ 0 , i.e., for which the core is mobile. Including the eigenvalue $\lambda=0$ we hence have

$$
\mathrm{N}_{\lambda}^{(1)}=\mathrm{g}+1
$$

distinct eigenvalues (i.e. relaxation times). We close this point by noting that here the set of nonvanishing eigenvalues differs from what is found for a "full" dendrimer. ${ }^{38}$ This is due to the fact that the core, which has a different functionality in the dendrimer than in the wedge, is involved in the motion.

(ii) I mmobile Core. The next group of motions we consider implies an immobile core. 27,38 In contrast to the previous case, here one can expect for the dendritic wedges the same set of relaxation times as for the dendrimers. The only difference will consist in the degeneracy of these modes. F or instance, if a bead next to the core is mobile, the degeneracy of the eigenvalues will be ( $f-2)$-fold, as compared to ( $f-1)$-fold for the "full" dendrimers. ${ }^{38}$ The reason is that one can choose as eigenmodes those in which most of the neighboring beads of the core and their descendents are immobile, so only two neighboring beads (and their subwedges) move against each other. ${ }^{27}$ These beads act as "roots". Now focusing on such one "root", one can pick for it exactly ( $f-2$ ) different partner "roots", by which one obtains a set of corresponding, $(f-2)$ linearly independent eigenmodes. It is then a simple matter to verify that the other eigenmodes of this class follow by a linear operation (a subtraction) from the members of the set. ${ }^{27}$

Thus, when the core is immobile, the problem turns (due to the symmetry of the system) into that of separated, mobile subwedges. On each subwedge the symmetrized equations of motion for the inner beads, eq A5, are not changed. Moreover, as we proceed to show, the eigenfunctions will have the form $\Phi_{k}{ }^{s}(j)$, so that eq A8 again holds. Furthermore, eq A6 for the peripheral beads also stays as before. What changes, however, is eq A7, which is the symmetrized equation of motion at the inner boundary. To fix the ideas let us 
consider the case that beads of the first generation are mobile, whereas the core is immobile. In this case eq A7 is replaced by 38

$$
\left(-\lambda_{k}\right) \Phi_{k}(1)+\left[f \Phi_{k}(1)-\sqrt{f-1} \Phi_{k}(2)\right]=0
$$

and eq $\mathrm{A} 5$ holds for $\mathrm{j}=1, \ldots, \mathrm{g}$. It is now a simple matter to verify that the functions

$$
\Phi_{\mathrm{k}}{ }^{\mathrm{s}}(\mathrm{j})=\sin \mathrm{j} \psi_{\mathrm{k}} \text { with } \mathrm{j}=1, \ldots, \mathrm{g}
$$

where the $\psi_{\mathrm{k}}$ fulfill

$$
\sin (g+1) \psi_{k}=\sqrt{f-1} \sin g \psi_{k}
$$

solve eqs $\mathrm{A} 6$ and $\mathrm{A} 13$ for $\lambda_{\mathrm{k}}$ given by eq $\mathrm{A} 8$.

Now we turn to show that the number of different eigenvalues which follow from eqs A8 and A15 is either $(\mathrm{g}-1)$ or $\mathrm{g}$. Since $\psi_{\mathrm{k}}=0$ leads to the trivial solution $\Phi_{\mathrm{k}}(\mathrm{j}) \equiv 0$ and since through eq A8 the eigenvalues $\lambda_{\mathrm{k}}$ are determined by $\cos \psi_{\mathrm{k}}$, we must restrict the phase shift $\psi_{\mathrm{k}}$ to an interval, e.g. to $0<\psi_{\mathrm{k}}<\pi$; all other $\psi_{\mathrm{k}}$ reproduce in eq A8 mod $\pi$ the same $\lambda_{\mathrm{k}}$.

Graphically, the number of solutions $\psi_{\mathrm{k}}$ of eq A15 corresponds to the number of crossings of the curves $y_{1}$ $=\sin (g+1) x$ and $y_{2}=\sqrt{f-1} \sin g x$ inside the interval $[0, \pi]$. Now $y_{2}$ crosses at $x_{m}=m \pi / g$ the $x$ axis and these crossings divide the interval $[0, \pi]$ into $g$ segments. Evidently, $\mathrm{y}_{1}\left(\mathrm{x}_{\mathrm{m}}\right) \neq 0$ for $1 \leq \mathrm{m} \leq(\mathrm{g}-1)$, and there is a change of sign between consecutive $y_{1}\left(x_{m}\right)$. It follows that within each internal segment $\mathrm{y}_{1}(\mathrm{x})$ changes sign once, whereas $\mathrm{y}_{2}(x)$ keeps its sign unchanged and equals zero at the segment's ends. Hence $y_{1}(x)$ and $y_{2}(x)$ cross each other inside each internal segment. The two external segments require more care, since for them $\mathrm{y}_{1}\left(\mathrm{x}_{0}\right)=0=\mathrm{y}_{1}\left(\mathrm{x}_{\mathrm{g}}\right)$. Now, in the segment $\left[\mathrm{x}_{\mathrm{g}-1}, \mathrm{x}_{\mathrm{g}}\right]$ the functions $y_{1}(x)$ and $y_{2}(x)$ have mainly different signs, the region of equal signs being concentrated close to $x_{g-1}$; this, together with $\mathrm{y}_{2}\left(\mathrm{x}_{\mathrm{g}-1}\right)=0$ and $\mathrm{y}_{1}\left(\mathrm{x}_{\mathrm{g}-1}\right) \neq 0$ again guarantees a crossing. The situation is different for $\left[0, x_{1}\right]$, where $y_{1}(x)$ and $y_{2}(x)$ have mainly equal signs. Then the existence of a crossing point depends on whether one has $y_{1}(x)>y_{2}(x)$ for $x$ close to zero or not, i.e., on the slopes of the curves at $x=0$; a crossing exists only for $(g+1)>\sqrt{f-1} g$. Summarizing, we find for $(g+1)>\sqrt{f-1} g$ a total of $g$ distinct solutions of eqs $A 8$ and $A 15$; otherwise the number of distinct solutions is $(g-1)$. Note that the condition $(g+1)>\sqrt{f-1} g$ is fulfilled only in a few cases, namely, for $f=3$ with $g=1$ and $g=2$ and for $f=4$ with $g=1$. For all other values of the system's parameters we find, on the basis of eq A8, $(g-1)$ eigenvalues whose eigenmodes are periodical functions of $j$.

Given that the linear system of equations given by eqs A5, A6, and A13 has $g$ solutions, for $(g+1) \leq$ $\sqrt{f-1} \mathrm{~g}$ one eigenmode must differ from eq A14. Indeed, one can verify readily that the eigenfunction ${ }^{38}$

$$
\Phi(\mathrm{j})=\sinh \mathrm{j} \psi
$$

fulfills eq $A 5$ for

$$
\Lambda=f-2 \sqrt{f-1} \cosh \psi
$$

It also fulfills eq $A 13$, whereas eq $A 6$ requires in addition that also

$$
\sinh (g+1) \psi=\sqrt{f-1} \sinh g \psi
$$

hol ds. It is easy to see (for instance, again by means of a simple drawing) that, apart from the trivial solution $\psi=0$, eq A18 has a single additional solution $\psi$ if and only if $(g+1) \leq \sqrt{f-1} g$. In this way we have in all cases $g$ different eigenvalues. Note that here one has two sorts of normal modes: The first sort is given by periodic-type modes in space, see eqs A8, A14, and A15, and is related to the internal relaxation inside the given wedge's subbranch. I $\mathrm{n}$ contrast, the second sort has an exponential-type normal mode in space, see eqs A16, A17, and A18, which corresponds to the motion of the whole subbranch with respect to the others (under the condition of an immobile core). Taking now into account also the ( $f-2)$-fold degeneracy discussed above, we obtain a total of $(f-2) g$ eigenvalues for modes where the core is immobile but where next neighbors to the core move.

We now turn to the general case which allows larger groups of noncore beads to stay immobile and let $\mathrm{n}$ denote (with $n<(g-1)$ ) the last generation in which all beads are immobile. One may note that the case $n$ $=0$ was just discussed. This last generation contains $(f-1)^{n}$ immobile beads, and we focus on a particular one, to which ( $f-1)$ mobile beads are attached. As before, the combination of ( $f-1)$ subwedges implies a $(f-2)$-fold degeneracy, so that the total degeneracy is now $(f-1)^{n}(f-2)$-fold, with $n \in\{1, \ldots, g-1\}$.

Now for $\Phi_{k}(j) \equiv 0$ (with $0 \leq j \leq n$ ) and $\Phi_{k}(n+1) \neq 0$, eq $A 5$ holds for $\mathrm{n}<\mathrm{j}<\mathrm{g}$, eq A6 stays unchanged, and eq $A 13$ is replaced by

$$
\left(-\lambda_{k}\right) \Phi_{k}(n+1)+\left[f \Phi_{k}(n+1)-\sqrt{f-1} \Phi_{k}(n+2)\right]=0
$$

This leads, by a simple reinterpretation of eqs A14 and $A 15$, to the following set of eigenfunctions:38

$$
\Phi_{k}{ }^{s}(j)=\sin (j-n) \psi_{k}
$$

where the eigenvalues are given by eq A8 and the $\psi_{\mathrm{k}}$ have to fulfill

$$
\sin (\mathrm{g}+1-\mathrm{n}) \psi_{\mathrm{k}}=\sqrt{\mathrm{f}-1} \sin (\mathrm{g}-\mathrm{n}) \psi_{\mathrm{k}}
$$

As before, eq A21 has in the interval $0<\psi_{\mathrm{k}}<\pi$ exactly $(g-n)$ distinct solutions if $(g-n+1)>$ $\sqrt{f-1}(g-n)$. Otherwise, when $(g-n+1) \leq \sqrt{f-1}(g-$ $n)$, there are $(g-n-1)$ distinct solutions of the form of eq A20 and one has an additional solution in the form of eqs A16 and A17, where now

$$
\Phi(\mathrm{j})=\sinh (\mathrm{j}-\mathrm{n}) \psi
$$

whose eigenvalue is given by eq A17. The additional condition corresponding to eq A18 reads now

$$
\sinh (\mathrm{g}-\mathrm{n}+1) \psi=\sqrt{\mathrm{f}-1} \sinh (\mathrm{g}-\mathrm{n}) \psi
$$

The last equation has an unique nontrivial solution if and only if $(g-n+1) \leq \sqrt{f-1}(g-n)$. All in all, taking into account the degeneracies, we find here a total of $(g-n)(f-2)(f-1)^{n}$ eigenvalues.

Finally, we consider the special case $n=(g-1)$, in which only the peripheral monomers are able to move. 
Here, given that $\Phi_{k}(g-1)=0$, we have directly from eq A6

$$
\left(-\lambda_{k}\right) \Phi_{k}(g)+\Phi_{k}(g)=0
$$

Equation $\mathrm{A} 24$ has the unique solution $\lambda=1$, which is $(f-1)^{g-1}(f-2)$-fold degenerate.

We now calculate $N_{\lambda}{ }^{(2)}$, the total number of eigenvalues (relaxation times) for all normal modes in which the core is immobile. Summarizing the discussion, and including also the case $n=0$, we have for each $n \in\{0$, $\ldots, g-1\}$ exactly $(g-n)$ distinct eigenvalues, which are each $(f-2)(f-1)^{n}$-fold degenerate. Hence

$$
\begin{aligned}
N_{\lambda}{ }^{(2)}=\sum_{n=0}^{g-1}(g-n)(f-2)(f-1)^{n} & = \\
& \frac{(f-1)^{g+1}-1}{f-2}-g-1=N_{w}-g-1
\end{aligned}
$$

where in the last equation we used eq 4.

Summing up now the number of eigenvalues found, we have from eqs A12 and A25

$$
\mathrm{N}_{\lambda}=\mathrm{N}_{\lambda}{ }^{(1)}+\mathrm{N}_{\lambda}^{(2)}=(\mathrm{g}+1)+\mathrm{N}_{\mathrm{w}}-\mathrm{g}-1=\mathrm{N}_{\mathrm{w}}
$$

which proves that we have indeed found all the eigenvalues (relaxation times). As it should be, $\mathrm{N}_{\lambda}$ equals $\mathrm{N}_{\mathrm{w}}$, the total number of monomers (beads) of the dendritic wedge.

\section{Appendix B}

Here we present the evaluation of the minimal nonvanishing eigenvalue (corresponding to the maximal relaxation time) of the dendritic wedge. It is easy to see from eq 5 that for both types of modes (which involve either a mobile or an immobile core) the eigenvalues given by eq 5 are bounded from below by $\lambda_{\min }{ }^{(\cos )}=$ $f-2 \sqrt{f-1}$, which for $f \geq 3$ (note that nontrivial dendrimers have $f \geq 3$ ) is al ways positive; furthermore $\lambda_{\text {min }}{ }^{(\cos )}$ does not depend on $\mathrm{g}$. As we proceed to show, in general there are further nonvanishing eigenvalues of the wedge, which lie lower than $\lambda_{\min }{ }^{\text {(cos); }}$ they stem from eq 8, where $\psi$ follows from eq 9.

Using the new variable $z=\exp \psi$, eq 9 reads $\left(z^{g+1}-\right.$ $\left.z^{-g^{-1}}\right) /\left(z^{g}-z^{-g}\right)=\sqrt{f-1}$, which we prefer to rewrite as

$$
z=\sqrt{f-1} \frac{1-z^{-2 g}}{1-z^{-2 g-2}}
$$

From the solution $z$ of eq $B 1$ the eigenvalue $\Lambda$ follows

$$
\Lambda=f-\sqrt{f-1}\left(z+z^{-1}\right)
$$

by rewritting eq 8 . For large $\mathrm{g}$ one can obtain $\mathrm{z}$ iteratively from eq B1. Evidently, a starting point $(g \rightarrow \infty)$ is $z^{(0)}=\sqrt{f-1}$, from which, with eq B2, follows

$$
\Lambda^{(0)}=f-\sqrt{f-1}(\sqrt{f-1}+1 / \sqrt{f-1})=0
$$

A better solution to $z$ (to order $z^{-2 g} \simeq(f-1)^{-g}$ ) is obtained by inserting $z^{(0)}$ into the right-hand side of eq B1; it leads to this order to

$$
\begin{aligned}
z^{(1)}=\sqrt{f-1}\left[1-(f-1)^{-g}+(f-1)^{-g-1}\right]= \\
\sqrt{f-1}\left[1-(f-2)(f-1)^{-g-1}\right]
\end{aligned}
$$

which, inserted into eq B2, gives to this order

$$
\Lambda^{(1)}=f-\sqrt{f-1}\left(z^{(1)}+1 / z^{(1)}\right)=(f-2)^{2} /(f-1)^{g+1}
$$

Note that $\Lambda^{(1)}$ decreases exponentially with $g$.

Furthermore for modes which involve immobile noncore beads, see eqs 8 and 11, one has an expression similar to $\Lambda^{(1)}$ for the corresponding eigenvalue, where $\mathrm{g}$ in eq $\mathrm{B} 5$ is replaced by $(\mathrm{g}-\mathrm{n})$ with $\mathrm{n} \in\{1, \ldots, \mathrm{g}-1\}$. Thus, it follows that the minimal, nonvanishing eigenvalue for the wedge, $\lambda_{\min }$, behaves for large $g$ as $^{38}$

$$
\lambda_{\min } \simeq \Lambda^{(1)}=\frac{(f-2)^{2}}{(f-1)^{(g+1)}}
$$

Physically, the smallest eigenvalue $\lambda_{\min }$ (the longest relaxation time) corresponds to a mode in which the largest dendritic branches move as a whole with respect to each other. 27,38

\section{References and Notes}

(1) Tomalia, D. A.; Naylor, A. M.; Goddard, W. A. Angew. Chem., Int. Ed. Engl. 1990, 29, 138.

(2) Moreno-Bondi, M. C.; Prelana, G.; Turro, N. J .; Tomalia, D. A. Macromolecules 1990, 23, 910.

(3) Hawker, C. J .; Fréchet, J . M. J . J . Am. Chem. Soc. 1990, 112, 7638.

(4) Hawker, C. J .; Fréchet, J . M. J . Macromolecules 1990, 23, 4726.

(5) NewKome, G. R. Advances in Dendritic Macromol ecules; J Al Press: London, 1996.

(6) Fréchet, J. M. J . Science 1994, 263, 1710.

(7) Yin. R.; Zhu, Y.; Tomalia, D. A. J . Am. Chem. Soc. 1998, 120, 2678.

(8) Percec, V.; Ahn, C.-H.; Ungar, G.; Yeardly, D. J . P.; Möller, M.; Sheiko, S. S. Nature 1998, 391, 161.

(9) J ahromi, S.; Coussens, B.; Meijerink, N.; Braam, A. W. M. J. Am. Chem. Soc. 1998, 120, 9753.

(10) J ahromi, S.; Litvinov, V.; Coussens, B. Macromolecules 2001, 34, 1013.

(11) Rouse, P. E. J . Chem. Phys. 1953, 21, 1272.

(12) Doi, M.; Edwards, S. R. The Theory of Polymer Dynamics; Clarendon: Oxford, U.K., 1986.

(13) Uppuluri, S.; Keinath, S. E.; Tomalia, D. A.; Dvornic, P. R. Macromolecules 1998, 31, 4498.

(14) J ahromi, S.; Palmen, J . H. M.; Steeman, P. A. M. Macromolecules 2000, 33, 577.

(15) Sommer, J.-U.; Blumen, A. J . Phys. A 1995, 28, 6669.

(16) Schiessel, H. Phys. Rev. E 1998, 57, 5775.

(17) Schiessel, H.; Friedrich, C.; Blumen, A. In Applications of Fractional Calculus in Physics; Hilfer, R., Ed.; World Scientific: Singapore, 2000; p 331.

(18) Ferry, J . D. Viscoelastic Properties of Polymers, 3rd ed.; Wiley: New York, 1980.

(19) Yamakawa, H. Modern Theory of Polymer Solutions; Harper and Row: New York, 1971.

(20) Ganazzoli, F.; La Ferla, R.; Terragni, G. Macromolecules 2000, 33, 6611.

(21) Ganazzoli, F.; La Ferla, R.; Raffaini, G. Macromolecules 2001 34, 4222.

(22) Graessley, W. W. Macromolecules 1980, 13, 372.

(23) de Gennes, P. G.; Hervet, H. J . Phys. (Paris) 1983, 44, L 351.

(24) Kloczkowski, A.; Mark, J . E.; Frisch, H. L. Macromolecules 1990, 23, 3481.

(25) Biswas, P.; Cherayil, B. J . J . Chem. Phys. 1994, 100, 3201.

(26) Boris, D.; Rubinstein, M. Macromolecules 1996, 29, 7251.

(27) Cai, C.; Chen, Z. Y. Macromolecules 1997, 30, 5104

(28) La Ferla, R. J . Chem. Phys. 1997, 106, 668.

(29) Chen, Z. Y.; Cai, C. Macromol ecules 1999, 32, 5423.

(30) Ganazzoli, F.; La Ferla, R. J . Chem. Phys. 2000, 113, 9288.

(31) Biswas, P.; Kant, R.; Blumen, A. Macromol. Theory Simul. 2000, 9, 56.

(32) Biswas, P.; Kant, R.; Blumen, A. J . Chem. Phys. 2001, 114 2430.

(33) Lescanec, R. L.; Muthukumar, M. Macromolecules 1990, 23, 2280. 
(34) Mansfield, M. L.; Klushin, L. I. Macromolecules 1993, 26, 4262.

(35) Murat, M.; Grest, G. S. Macromolecules 1996, 29, 1278.

(36) Chen, Z. Y.; Cui, S.-M. Macromolecules 1996, 29, 7943.

(37) Lyulin, A. V.; Davies, G. R.; Adolf, D. B. Macromol ecules 2000 33, 3294.

(38) Gotlib, Yu. Ya; Markelov, D. A. Vysokomol. Soedin., Ser. A 2002, in press.

(39) Press, W. H.; Flannery, B. P.; Teukolsky, S. A.; Vetterling, W. T. Numerical Recipes in C. The Art of Scientific Computing; Cambridge University Press: New York, 1988.

(40) Gotlib, Yu. Ya.; Darinsky, A. A.; Svetlov, Yu. E. Physical Kinetics of Macromolecules; Khimiya: Leningrad, Russia, 1986.

(41) Sommer, J.-U.J . Chem. Phys. 1991, 95, 1316.

(42) Mooney, M. J . Polym. Sci. 1959, 34, 599.
(43) Gurtovenko, A. A.; Blumen, A. J . Chem. Phys. 2001, 115, 4924.

(44) Gurtovenko, A. A.; Blumen, A. Macromolecules 2002, 35, 3288.

(45) Gurtovenko, A. A.; Gotlib, Yu. Ya. J . Chem. Phys. 2001, 115, 6785.

(46) Gurtovenko, A. A.; Gotlib, Yu. Ya. Macromolecules 1998, 31, 5756.

(47) Gurtovenko, A. A.; Gotlib, Yu. Ya. Macromolecules 2000, 33, 6578.

(48) Gotlib, Yu. Ya.; Gurtovenko, A. A. Macromol. Theory Simul. 2000, 9, 407.

(49) Gurtovenko, A. A.; Gotlib, Yu. Ya. Macromol. Theory Simul. 2000, 9, 416 .

MA0204507 